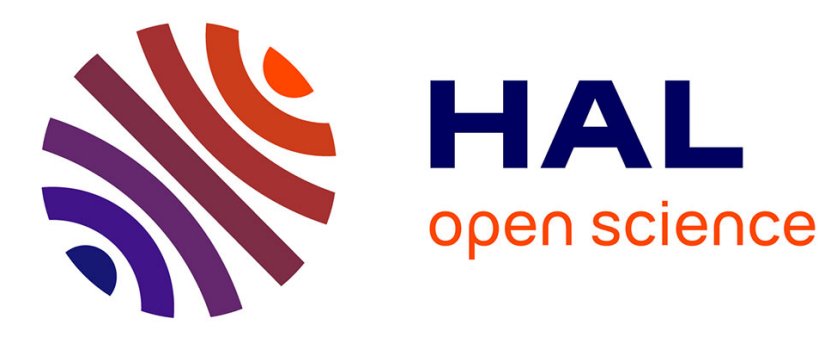

\title{
On the homogeneity at infinity of the stationary probability for an affine random walk
}

\author{
Yves Guivarc'H, Emile Le Page
}

\section{To cite this version:}

Yves Guivarc'H, Emile Le Page. On the homogeneity at infinity of the stationary probability for an affine random walk. Siddhartha Bhattacharya, Tarun Das, Anish Ghosh and Riddhi Shah. Recent trends in ergodic theory and dynamical systems, 631, American Mathematical Society, pp.119-130, 2015, Contemporary Mathematics, 978-1-4704-0931-9. hal-00868944

\section{HAL Id: hal-00868944 https://hal.science/hal-00868944}

Submitted on 2 Oct 2013

HAL is a multi-disciplinary open access archive for the deposit and dissemination of scientific research documents, whether they are published or not. The documents may come from teaching and research institutions in France or abroad, or from public or private research centers.
L'archive ouverte pluridisciplinaire HAL, est destinée au dépôt et à la diffusion de documents scientifiques de niveau recherche, publiés ou non, émanant des établissements d'enseignement et de recherche français ou étrangers, des laboratoires publics ou privés. 


\title{
On the homogeneity at infinity of the stationary probability for an affine random walk.
}

\author{
by Y. Guivarc'h, E. Le Page
}

\begin{abstract}
$\underline{\text { Abstract }}$
We consider an affine random walk on $\mathbb{R}$. We assume the existence of a stationary probability $\nu$ on $\mathbb{R}$ and we describe the shape at infinity of $\nu$, if $\nu$ has unbounded support. We discuss the connections of the result with geometrical or probabilistic problems.
\end{abstract}

\section{$\underline{I}$ - Introduction}

Let $G$ be the affine group of the line. For $g \in G, x \in \mathbb{R}$, we write $g x=a(g) x+b(g)$ with $a(g) \in \mathbb{R}^{*}, b(g) \in \mathbb{R}$. Let $\mu$ be a probability on $G$. We denote by $P$ the Markov operator on $\mathbb{R}$ defined by $P \varphi(x)=\int \varphi(g x) \mu(d g)$ where $\varphi$ is a bounded Borel function. Our hypothesis $H_{\mu}$ is stated below and we observe that $H_{\mu}(1)$ and $H_{\mu}(2)$ imply that $P$ has a unique stationary probability $\nu$ (see [16]) ; if $H_{\mu}(4)$ is also valid, then supp $\nu$ is unbounded. Here we are interested in the "shape at infinity" of $\nu$; we will show that for some $\alpha>0$, the quantities $|t|^{\alpha} \nu[t, \infty)$ and $|t|^{\alpha} \nu(-\infty, t]$ have limits at infinity, we discuss their positivity and we illustrate the possible uses of this result by two corollaries in two different contexts. This "homogeneity at infinity" of $\nu$ plays an essential role in extreme value theory (see [19]), for random variables associated with the Markov chain $X_{n}^{x}$ with kernel $P$ on $\mathbb{R}$. Also, for random walk in a random medium on $\mathbb{Z}$ (see [21]) the slow diffusion property is closely related to this homogeneity (see [6], [17]). Furthermore the construction of $\nu$ given here provides a natural construction of a large class of heavy tailed measures which generates "anomalous" random walks on the additive group $\mathbb{R}$. This class of measures appears now to be of great interest from the physical point of view (see [2]). In the geometrical context of excursions of geodesic flows on manifolds of negative curvature the "logarithm law" is well known (see [22], [18]), and we will discuss analogous properties for the Markov chain $X_{n}^{x}$.

We assume that $\mu$ satisfies the following set of conditions $H_{\mu}$.

$H_{\mu}(1): \int(|\ell n| a(g)||+\mid(\ln |b(g)| \mid)) \mu(d g)<\infty$.

$H_{\mu}(2)$ : For some $\alpha>0 \int|a(g)|^{\alpha} \mu(d g)=1$.

$H_{\mu}(3): \int|a(g)|^{\alpha} \ell n|a(g)| \mu(d g)<\infty, \int|b(g)|^{\alpha} \mu(d g)<\infty$.

$H_{\mu}(4)$ : The elements of supp $\mu$ have no common fixed point in $\mathbb{R}$.

$H_{\mu}(5)$ : The set $\{\ln |a(g)| ; g \in$ supp $\mu\}$ generates a dense subgroup of $\mathbb{R}$.

Then we have the

\section{Theorem 1}

Assume that $\mu$ satisfies $H_{\mu}$. Then

1) There exists $c_{+} \geq 0, c_{-} \geq 0$ such that $\lim _{t \rightarrow \infty}|t|^{\alpha} \nu(t, \infty)=c_{+}, \lim _{t \rightarrow-\infty}|t|^{\alpha} \nu(-\infty, t)=c_{-}$.

Moreover $c=c_{+}+c_{-}>0$.

2) If $\mu\{g \in G ; a(g)<0\}>0$, then $c_{+}=c_{-}>0$. 
3) If $\mu\{g \in G ; a(g)>0\}=1$, then $c_{+}>0$ (resp $c_{-}>0$ ) if and only if the action of supp $\mu$ on $\mathbb{R}$ has no invariant half-line of the form $]-\infty, k]$, (resp $[k, \infty[)$.

4) If $\mu\{g \in G ; a(g)<0\}>0$, then supp $\nu=\mathbb{R}$. Otherwise the set supp $\nu$ is a half-line if and only if supp $\mu$ preserves a half-line of the same form.

We denote by $\mathbb{P}$ the product probability $\mu^{\otimes \mathbb{N}}$ on $\Omega=G^{\mathbb{N}}$, where $\mathbb{N}$ is the set of positive integers. For $\omega \in \Omega$, we write $g_{k}=\left(a\left(g_{k}\right), b\left(g_{k}\right)\right)=\left(a_{k}, b_{k}\right)$. Then $X_{n}^{x}$ satisfies the stochastic recursion :

$X_{n}^{x}=a_{n} X_{n-1}^{x}+b_{n}, X_{0}^{x}=x$.

The Markov chain $X_{n}^{x}$ on $\mathbb{R}$ will be called "affine random walk". It is well known that, for the existence and uniqueness of the stationary measure $\nu$, it is sufficient to assume $H_{\mu}(1)$ and $\int \ln |a(g)| \mu(d g)<0$; then $X_{n}^{x}$ converges in law to $R=\sum_{1}^{\infty} a_{1} \ldots a_{k-1} b_{k}$ and the law of $R$ is $\nu$. Also if $\int\left(|a(g)|^{\beta}+|b(g)|^{\beta}\right) \mu(d g)<\infty$ for some $\beta>0$, then $\int|x|^{\beta} d \nu(x)<\infty$. We observe that $R$ can be interpreted as the sum of a "random geometric series", hence its interest for collective risk theory ([19]).

The validity of 1) and 2) was proved in [10], [16] ; in particular implicit expression for $c_{+}, c_{-}$ were given in [10] and the relation $c_{+}+c_{-}>0$ was obtained. Here we restrict our study to 3 ) and 4), a result which is new under hypothesis $H_{\mu}$. A different proof was sketched in [12], where a survey of the multidimensional situation was also given. We observe that the main difficulty of the proof occurs when supp $\mu$ do not preserve a half line and $a(g)>0$ $\mu-a . e$; in this case we have $c_{+}>0, c_{-}>0$. If supp $\mu$ has compact support a short complex analytic proof of this fact, depending of a Lemma of E. Landau, well known in analytic number theory, is given in [11] (see also [5]).

We recall that Fréchet's law with parameter $\gamma$ is the probability $\Phi_{\alpha}^{\gamma}$ on $\mathbb{R}_{+}$given by $\Phi_{\alpha}^{\gamma}(0, t)=e^{-\gamma t^{-\alpha}}$ where $\gamma>0, \alpha>0$. This family of laws is one of the three families of max-infinitely divisible laws of extreme value theory ([9], [19]). The following is shown in [14].

\section{Corollary 2}

For $x \in \mathbb{R}$ we denote

$$
M_{n}^{x}=\sup \left\{\left|X_{k}^{x}\right| ; 1 \leq k \leq n\right\},+M_{n}^{x}=\sup \left\{X_{n}^{x} ; 1 \leq k \leq n\right\} .
$$

Then the sequence $n^{-\alpha} M_{n}^{x}\left(\right.$ resp $\left.n^{-\alpha}+M_{n}^{x}\right)$ converges in $\mathbb{P}$-law to $\Phi_{\alpha}^{c \theta}$ with $0<\theta<1$ (resp $\Phi_{\alpha}^{c_{+} \theta_{+}}$with $0<\theta_{+}<1$, if $\left.c_{+}>0\right)$.

Closely related properties have been intensively studied in the context of extreme value theory (see [19]). The positive number $\theta$ is the so-called extremal index of the stochastic process $X_{n}^{x}$; its inverse $\theta^{-1}$ gives a measure of the clustering of the exceptionally large values of the process. If the random variables $X_{n}^{x}$ were i.i.d. with law $\nu$, one would have $\theta=1$ (see [9]). If $a(g)>0, b(g)>0$ for $g \in \operatorname{supp} \mu$, the above corollary is proved in [15]. It is also known (see [13]) that, under hypothesis $H_{\mu}$, the normalized Birkhoff sum of $X_{n}^{x}$ 
converges in law to a stable law of index $\alpha$ if $\alpha<2$. As mentioned in ([3], remark 4.8), this convergence is a consequence of extreme value properties of $X_{n}^{x}$, at least for $\alpha<1$. The analysis of random walk in a random medium on $\mathbb{Z}$ developed in [6] is closely related to such properties for the sojourn time of the particle at a site in $\mathbb{Z}$, instead of its hitting time as in [17], where Birkhoff sums as above played a dominant role.

The following logarithm law is an easy consequence of Corollary 2.

\section{Corollary 3}

For any $x \in \mathbb{R}$, we have the following $\mathbb{P}$ - a.e convergences :

$$
\limsup _{n \rightarrow \infty} \frac{\ell n\left|X_{n}^{x}\right|}{\ln (n)}=\frac{1}{\alpha}, \limsup _{n \rightarrow \infty} \frac{\ln ^{+}\left(X_{n}^{x}\right)}{\ln (n)}=\frac{1}{\alpha} \text { if } c_{+}>0 .
$$

The so-called "logarithm law" for excursions of geodesic flow around the cusps on hyperbolic manifolds was proved in [22] and extended to more general situations in [18]. It was observed in [20] that in case of the modular surface, it is a simple consequence of Fréchet's law for geodesic flow which follows from already known extreme value properties of the continuous fraction expansion of a number $x$ uniformly distributed on $[0,1]$ (see [8]).

\section{II - Calculation of invariant measures on $\mathbb{R}$ in a special case}

The Lie algebra of $G$ is generated by the vector fields $X=a \frac{\partial}{\partial a}, Y=\frac{\partial}{\partial b}$. We consider the convolution semi-group of probability measures on $G$ with infinitesimal generator $D=$ $X^{2}+Y^{2}-(\beta+1) X$. This operator is elliptic and we denote by $p^{t}(t \geq 0)$ the associated semi-group of probability measures.

We have $\int \ln a(g) p^{t}(d g)=-t(\beta+1)$ in particular $\int \ln a(g) p(d g)$ is negative if $\beta>-1$, hence $p^{t}$ has a stationary probability $\nu$ on $\mathbb{R}$ in this case. We consider more generally, for any $\beta$, the action of $p^{t}$ and $X, Y, D$ on positive measures of the form $\nu=f(x) d x$ on the line. We denote by $X^{*}, Y^{*}, D^{*}$ the operators adjoint to $X, Y, D$. Then the extremal solutions of the equation $D^{*} f=0(f \geq 0)$ are described by the

\section{Proposition 1}

With the above notations, the equation $D^{*} f=0$ has the following normalized extremal solutions :

$$
\begin{aligned}
& \quad \begin{array}{l}
\beta \geq-1: f(x)=\left(1+x^{2}\right)^{-(1+\beta / 2)} \\
\beta<-1: f_{+}(x)=\left(1+x^{2}\right)^{-(1+\beta / 2)} \int_{-\infty}^{x}\left(1+t^{2}\right)^{\beta / 2} d t,
\end{array} \\
& \quad \text { and } f_{-}(x)=\left(1+x^{2}\right)^{-(1+\beta / 2)} \int_{x}^{\infty}\left(1+t^{2}\right)^{\beta / 2} d t . \\
& \text { If } \beta>-1 \text {, then } \int f(x) d x<\infty \text {. If } \beta \leq-1 \text { then } \int f(x) d x=\int f_{+}(x) d x=\int_{-} f(x) d x=\infty .
\end{aligned}
$$

\section{$\underline{\text { Proof }}$}

We calculate the action of $X, Y$ on the measure $\nu=f d x$ as follows.

Since $d x$ is translation-invariant and the action of the one parameter group $x \rightarrow x+b$ is by translation we get $Y^{*} f=-f^{\prime}$.

Since $X \varphi(x)=x \varphi^{\prime}(x)$, we get also $X^{*} f(x)=-(x f(x))^{\prime}$. It follows $D^{*} f(x)=\left(x(x f)^{\prime}\right)^{\prime}+$ 
$f^{\prime \prime}+(\beta+1)(x f)^{\prime}$, so that the equation $D^{*} f=0$ implies :

$x(x f)^{\prime}+f^{\prime}(x)+(\beta+1)(x f)=k$,

for a certain constant $k$, i.e :

$\left(1+x^{2}\right) f^{\prime}+(\beta+2)(x f)=k$.

With $u(x)=\left(1+x^{2}\right)^{-(1+\beta / 2)}$ we have $\left(1+x^{2}\right) u^{\prime}(x)+(\beta+2) x u(x)=0$, hence the above differential equation has the solutions : $f=u(d+k v)$ with $v(x)=\int_{0}^{x}\left(1+t^{2}\right)^{\beta / 2} d t$ and $d$ is a constant.

For $\beta \geq-1$, we have $\lim _{x \rightarrow \infty} v(x)=\infty$, hence the condition $f \geq 0$ implies $k=0$. In this case the equation $\stackrel{x \rightarrow \infty}{D^{*} f}=0$ has only positive extremal solutions of the form $f(x)=$ $d\left(1+x^{2}\right)^{-(1+\beta / 2)}$. For $\beta=0, D$ is the hyperbolic Laplacian and we recover the Cauchy law on $\mathbb{R}$ with density $\frac{1}{\pi} \frac{1}{1+x^{2}}$. For $\beta>-1$, we get a probability law with density proportional to $\left(1+x^{2}\right)^{-(1+\beta / 2)}$.

We verify that for $\beta<-1$, the equation $D^{*} f=0$ has two basic extremal solutions :

$$
\begin{aligned}
& f_{+}(x)=\left(1+x^{2}\right)^{-(1+\beta / 2)} \int_{-\infty}^{x}\left(1+t^{2}\right)^{\beta / 2} d t, \\
& f_{-}(x)=\left(1+x^{2}\right)^{-(1+\beta / 2)} \int_{x}^{\infty}\left(1+t^{2}\right)^{\beta / 2} d t .
\end{aligned}
$$

The measure $\nu$ corresponding to $f_{+}$has infinite mass and satisfies :

$$
\lim _{t \rightarrow-\infty}|t|^{2+\beta} \nu(-\infty, t)=c->0
$$

At $+\infty f_{+}(x)$ is asymptotic to $c_{+} x^{-1}$ with $c_{+}>0$. Analogous properties are valid fo $f_{-}$. Also, at $\infty, f(x)$ is asymptotic to $c|x|^{-1}(c>0)$

\section{Remark}

The case $\beta>-1$ corresponds to the situation of the theorem with $\alpha=\beta+1$.

The case $\beta=-1$ corresponds to the (critical) situation of [1], [4]. Then the unique basic extremal solution behaves at infinity like multiplicative Lebesgue measure on $\mathbb{R}^{*}$.

The situation $\beta<-1$, with two extremal solutions, corresponds to a so-called phase transition in P.D.E theory, for example in the context of non linear Schroëdinger equations.

\section{III - Proof of theorem 1}

The proofs of 1) and 2) in [10] are based on the first renewal equation in Lemma 1 below. A delicate point in [10] for the use of the renewal theorem (see [7]) is solved by replacing ${ }^{\alpha} f(t)=e^{\alpha t} f(t)$ by a related directly Riemann-integrable function. Here we give only the proofs of 3 ) and 4). We will now assume $\mu\{g ; a(g)>0\}=1$ and we will only study the non vanishing of $c_{+}$. To do that we need some preliminary notations and results.

Let $T$ be the stopping time on $\Omega$ defined by :

$$
T=\left\{n \geq 1 ; g_{1} g_{2} \cdots g_{n} \in G_{+}\right\}, T=\infty \text { if }\left\{n \geq 1 ; g_{1} g_{2} \cdots g_{n} \in G_{+}\right\}=\phi,
$$
where $G_{+}=\{b(g)>0\}$.

We denote by $\bar{\mu}$ the probability on the additive group $\mathbb{R}$ given by $\bar{\mu}(A)=\mu\{\ln a(g) \in A\}$

Moreover we denote by $\mu_{T}$ the positive measure on $\mathbb{R}$ defined by :

$$
\mu_{T}(A)=\mathbb{P}\left\{T<+\infty ; \ln \left(a_{1} a_{2} \cdots a_{T}\right) \in A\right\},
$$


where $A$ is a Borel subset of $\mathbb{R}$. We have $\mu_{T}(\mathbb{R})=\mathbb{P}(T<+\infty) \leq 1$, and we denote by $\mu_{T}^{n}$ the $n^{\text {th }}$ convolution power of $\mu_{T}$ on the additive group $\mathbb{R}$. Define $f$ by

$$
f(t)=\mathbb{P}\left\{R>e^{t}\right\}=\nu(] e^{t},+\infty[) \quad t \in \mathbb{R},
$$

and write $R_{n}=\sum_{k=1}^{n} a_{1} a_{2} \cdots a_{k-1} b_{k}, S_{n}=\sum_{k=1}^{n} \ln \left(a_{k}\right)$.

Then we have the :

\section{Lemma 1}

1) For every real $t$, we have $f(t)=\bar{\mu} * f(t)+f_{1}(t)=\mu_{T} * f(t)+h_{1}(t)$ where :

$$
\left.\left.f_{1}(t)=\mathbb{P}\left\{R-b_{1}>e^{t}\right\}-\mathbb{P}\left\{\mathbb{R}>e^{t}\right\}, h_{1}(t)=\mathbb{E}\left\{1_{[T<+\infty]} \nu(] e^{-S_{T}}\left(e^{t}-R_{T}\right), e^{t-S_{T}}\right]\right)\right\}
$$

2) For every real $t$, we have $f(t)=\sum_{n=0}^{+\infty} \mu_{T}^{n} * h_{1}(t)=\sum_{n=0}^{\infty} \mu^{n} * f_{1}(t)$.

If $p$ is a bounded measure on $\mathbb{R}$ and $\varphi$ is a positive Borel function, we write

$$
p * \varphi(t)=\int \varphi(t-x) p(d x), \quad t \in \mathbb{R} .
$$

We denote by ${ }^{\alpha} \mu$, the probability measure on $G$ defined by : ${ }^{\alpha} \mu(d g)=a^{\alpha}(g) \mu(d g)$.

We define the probability ${ }^{\alpha} \mathbb{P}$ on $G^{\mathbb{N}}$ by ${ }^{\alpha} \mathbb{P}={ }^{\alpha} \mu^{\otimes \mathbb{N}}$ and we write ${ }^{\alpha} \mathbb{E}$ for the corresponding expectation.

The measure ${ }^{\alpha} \mu_{T}$ on $\mathbb{R}$ is defined by ${ }^{\alpha} \mu_{T}(A)={ }^{\alpha} \mathbb{E}\left(1_{A}\left(\ln \left(a_{1} \cdots a_{T}\right)\right)\right.$, and we write ${ }^{\alpha} h_{1}(t)=e^{\alpha t} h_{1}(t) \quad t \in \mathbb{R}$.

Then from lemma 1 we get :

\section{Lemma 2}

For every real $t$ we have ${ }^{\alpha} f(t)=\sum_{n=0}^{+\infty} \alpha \mu_{T} *^{\alpha} h_{1}(t)$

Now we are going to study some properties of $T$ and $\ln \left(a_{1} a_{2} \cdots a_{T}\right)$ under ${ }^{\alpha} \mathbb{P}$. For that purpose we consider the new random variables $g_{i}^{\prime}(i \geq 1)$ defined by $g_{i}^{\prime}=\left(a_{i}^{-1}, b_{i} a_{i}^{-1}\right)$. Under ${ }^{\alpha} \mathbb{P}$, there random variables are i.i.d with law ${ }^{\alpha} \mu^{\prime}$. We have :

$$
g_{n}^{\prime} g_{n-1}^{\prime} \cdots g_{1}^{\prime}=\left(\left(a_{1} a_{2} \cdots a_{n}\right)^{-1}, R_{n}\left(a_{1} \cdots a_{n}\right)^{-1}\right),
$$

hence for $T^{\prime}=\operatorname{Inf}\left\{n ; g_{n}^{\prime} g_{n-1}^{\prime} \cdots g_{1}^{\prime} \in G_{+}\right\}$we have $T^{\prime}=T$. It follows that $T$ can be interpreted as the entrance time in $\left.\mathbb{R}_{+}=\right] 0, \infty$ [ of the affine random walk on $\mathbb{R}$ defined by ${ }^{\alpha} \mu^{\prime}$, starting from 0 . We denote by ${ }^{\alpha} Q$ the Markov kernel of this affine random walk, and for $p \in \mathbb{R}$ we write $p_{n}=g_{n}^{\prime} g_{n-1}^{\prime} \cdots g_{1}^{\prime} p$.

\section{Lemma 3}

1) There exists a unique probability measure ${ }^{\alpha} \nu^{\prime}$ on $\mathbb{R}$ such that ${ }^{\alpha} Q\left({ }^{\alpha} \nu^{\prime}\right)={ }^{\alpha} \nu^{\prime}$. The probability ${ }^{\alpha} \nu^{\prime}$ has no atoms.

2) If $\left.{ }^{\alpha} \nu^{\prime}(] 0,+\infty\right]>0$ then $0<{ }^{\alpha} \mathbb{E}\left(T^{\prime}\right)<\infty$.

Now we complete the proof of Theorem 1 using the above Lemmas.

For assertion 3, there are two cases.

$\left.\left.\underline{\text { First case }}{ }^{\alpha} \nu^{\prime}(] 0,+\infty\right]\right)>0$. 
Then by Lemma 3 and the observation before Lemma $3,{ }^{\alpha} \mathbb{E}(T)={ }^{\alpha} \mathbb{E}\left(T^{\prime}\right)<\infty,{ }^{\alpha} \mu_{T}(\mathbb{R})=1$. By Wald's lemma (see [7]), since $T^{\prime}<\infty^{\alpha} \mathbb{P}-$ a.e :

${ }^{\alpha} \mathbb{E}\left\{\ln \left(a_{1} a_{2} \cdots a_{T}\right)={ }^{\alpha} \mathbb{E}\left(\ell n\left(a_{1}\right)\right)^{\alpha} \mathbb{E}(T)\right.$

where ${ }^{\alpha} \mathbb{E}\left(\ell n\left(a_{1}\right)\right)=\mathbb{E}\left(a_{1}^{\alpha} \ell n\left(a_{1}\right)\right)$ is finite and positive, hence ${ }^{\alpha} \mathbb{E}\left(S_{T}\right)$ is finite and positive. Assume $c_{+}=0$, hence $\lim _{t \rightarrow \infty}{ }^{\alpha} f(t)=0$. Then, if we denote by ${ }^{\alpha} h_{1, L}(L>0)$ the function $t \rightarrow{ }^{\alpha} h_{1}(t) 1_{[-L, L]}(t)$, we have using Lemma 2 and Proposition A below : for every $L>0$,

$$
0=\lim _{t \rightarrow+\infty} \frac{1}{t} \int_{0}^{t} \sum_{n=0}^{+\infty} \alpha \mu_{T}^{n} *{ }^{\alpha} h_{1, L}(s) d s=\frac{1}{\alpha \mathbb{E}\left(\ln \left(a_{1}\right)\right)^{\alpha} \mathbb{E}(T)} \int_{-L}^{L}{ }^{\alpha} h_{1}(s) d s,
$$

hence $0=\int_{\mathbb{R}}{ }^{\alpha} h_{1}(s) d s$. Since ${ }^{\alpha} h_{1}$ and $h_{1}$ are non negative we get $h_{1}=0$ a.e, hence Lemma 1 implies $f(t)=0, d t-a . e$.

We conclude that for almost every real $s$ :

$$
f(s)=\mathbb{P}\left(R>e^{s}\right)=0,
$$

and so $\mathbb{P}(R \leq 0)=1$, hence supp $\nu \subset]-\infty, 0]$. It follows that supp $\mu$ preserves an interval $\left(-\infty, v_{0}\right)$ with $v_{0} \leq 0$.

\section{Second case ${ }^{\alpha} \nu^{\prime}(] 0,+\infty[)=0$.}

Denote by $v_{0} \leq 0$ the upper bound of the support of the probability ${ }^{\alpha} \nu^{\prime}$. Then by the stationarity property of ${ }^{\alpha} \nu^{\prime}$ we can write that for every $n \geq 1$ :

${ }^{\alpha} \mathbb{P}\left\{g_{n}^{\prime} g_{n-1}^{\prime} \cdots g_{1}^{\prime} v_{0} \leq v_{0}\right\}=1, \quad 1=\mathbb{E}\left(a_{1}^{\alpha} \cdots a_{n}^{\alpha} 1_{\left\{v_{0}+R_{n} \leq a_{1} \cdots a_{n} v_{0}\right\}}\right)$,

which implies that for every integer $n \geq 1, \mathbb{P}\left(R_{n} \leq-v_{0}\right)=1$ since $\mathbb{E}\left(a_{1}^{\alpha}\right)=1$. Since $R_{n}$ converges $\mathbb{P}$ - a.e to $R$ we have $\mathbb{P}\left(R \leq-v_{0}\right)=1$ hence $c_{+}=0$.

In conclusion we see that $c_{+}=0$ if and only if the upper bound of supp $\nu$ is finite i.e if supp $\mu$ preserves an interval $\left.]-\infty,-v_{0}\right]$.

In order to show assertion 4 we will distinguish the 2 cases $c_{+}>0, c_{-}=0, c_{+}>0$, and $c_{-}>0$. We observe that supp $\nu$ is invariant under supp $\mu$ and condition $\int \ln (a(g)) d \mu(g)<0$ implies that for some $g \in(\text { supp } \mu)^{2}$ we have $0<a(g)<1$. Also the complement of supp $\nu$ is invariant under $(\text { supp } \mu)^{-1}$. We denote by $T_{\mu}$ the closed subsemigroup of $G$ generated by supp $\mu$, and by $\Delta \subset \mathbb{R}$ the closure of the set of attractive fixed points of the elements of $T_{\mu}$. We observe that $T_{\mu} \Delta \subset \Delta$. Since for any $x \in \Delta$ the law of $g_{n} \cdots g_{1} x$ is supported by $\Delta$ and converges to $\nu$, we obtain that $\Delta \supset \operatorname{supp} \nu$. Since the attractive fixed points of $T_{\mu}$ belong to supp $\nu$, we conclude that $\Delta=$ supp $\nu$. Then, for any open interval $I=[a, b] \subset \mathbb{R}, n<0$, $g^{n}(I)$ is an interval of length $a^{n}(g)(b-a)$ which converges to $+\infty,-\infty$ or $\mathbb{R}$, depending of the relative positions of $I$ and the fixed point $x_{0}$ of $g$. If $c_{+}>0$ and $c_{-}=0$, then from above supp $\mu$ preserves the interval $[\tau, \infty[$ with $\tau=\operatorname{Inf}($ supp $\nu)$. Since $\Delta=$ supp $\nu$ we can choose $g \in(\text { supp } \mu)^{2}$ such that its fixed point $x_{0} \in$ supp $\nu$ is arbitrary close to $\tau$, and in particular $\tau \leq x_{0}<a$. If $\left.I \subset\right] \tau, \infty\left[\right.$ satisfies $\nu(I)=0$ then $\nu\left(g^{n}(I)\right)=0$ for $n<0$; since the length of the interval $g^{n}(I)$ is $a^{n}(g)(b-a)$ and $\lim _{n \rightarrow-\infty} a^{n}(g)=\infty$ this contradicts $c_{+}>0$.

If $c_{+}>0, c_{-}>0$ the same argument is valid for any interval $I$ with $\nu(I)=0$.

We now give the proofs of the above lemmas. 


\section{Proof of Lemma 1}

1) Denote $R^{n}=\sum_{k=n}^{+\infty} a_{n+1} \cdots a_{k} b_{k+1}$. Under $\mathbb{P}$ the law of $R_{n}$ is $\nu$ and moreover $R^{n}$ is independant of the random variables $g_{i}(1 \leq i \leq n)$.

The formula $R=R_{n}+a_{1} \cdots a_{n} R^{n}$ gives $R-b_{1}=a_{1} R^{1}$, hence :

$$
\mathbb{P}\left\{R-b_{1}>e^{t}\right\}=\mathbb{P}\left\{R^{1}>e^{t} a_{1}^{-1}\right\}=\mu * f(t), \quad f(t)=\mu * f(t)+f_{1}(t)
$$

We have also from above

$$
\begin{aligned}
& \left\{R>e^{t}\right\}=\left\{R_{T}+a_{1} a_{2} \cdots a_{\tau} R^{T}>e^{t}, T<\infty\right\} \\
& =\left\{R^{T}>e^{t-\ell n\left(a_{1} a_{2} \cdots a_{T}\right)} ; T<\infty\right\} U\left\{e^{t-\ell n\left(a_{1} a_{2} \cdots a_{T}\right)}<R^{T} \leq e^{t-\ell n\left(a_{1} a_{2} \cdots a_{T}\right)} ; T<\infty\right\}
\end{aligned}
$$

Using the fact that $T$ is a stopping time we have

$$
f(t)=\mathbb{P}\left\{R>e^{t}\right\}=\mathbb{P}\left\{R>e^{t}, T<\infty\right\}=\mu_{T} * f(t)+h_{1}(t)
$$

where $\left.h_{1}(t)=\mathbb{E}\left(1_{\{T<\infty\}} \nu\right] e^{t-\ell n\left(a_{1} \cdots a_{T}\right)}-R_{T}, e^{t-\ell n\left(a_{1} \cdots a_{T}\right)}\right]$,

It follows:

$$
f=\sum_{k=0}^{n} \bar{\mu}^{k} * f_{1}+\bar{\mu}^{n+1} * f
$$

where $\bar{\mu}^{n+1} * f(t)=\mathbb{P}\left\{R>e^{t}\left(a_{1} \cdots a_{n+1}\right)^{-1}\right\}$. The condition $\mathbb{E}\left(\ell n\left(a_{1}\right)\right)<0$ implies the $\mathbb{P}$ - a.e convergence of $\left(a_{1} \cdots a_{n+1}\right)^{-1}$ to $\infty$, hence $\lim _{n \rightarrow \infty} \bar{\mu}^{n+1} * f(t)=0$. The first part of the formula follows.

2) From above we deduce that for every integer $n$ and $t \in \mathbb{R}$.

$$
f(t)=\sum_{j=0}^{n} \mu_{T}^{j} * h_{1}(t)+\mu_{T}^{n+1} * f(t) .
$$

We now prove that $\quad \lim _{n \rightarrow+\infty} \mu_{T}^{n+1} * f_{1}(t)=0$.

There are two cases

We have

Case 1) $\mathbb{P}(T<\infty)<1$

$0 \leq \mu_{T}^{n+1} * f(t) \leq(\mathbb{P}(T<\infty))^{n}$

hence $\lim _{n \rightarrow \infty} \mu_{T}^{n+1} * f(t)=0$.

Case 2) $\mathbb{P}(T<\infty)=1$

Define the shift $\theta$ on $\Omega$ by $\theta(\omega)=\left(g_{i+1}(\omega), i \geq 1\right)$ where $\omega=\left(g_{i}(\omega), i \geq 1\right)$ and consider the sequence $\left(T_{n}(\omega)\right)_{n \geq 1}$ of random times defined $\mathbb{P}-$ a.e by $T_{n+1}=T_{0} \theta^{T_{n}}, \quad T_{1}=T$. Under $\mathbb{P}$ the sequence of random variables $\left[\left(T_{1}, S_{T_{1}}\right), \cdots,\left(T_{n+1}-T_{n}, S_{T_{n+1}}-S_{T_{n}}\right)\right]$, is i.i.d and the law of $S_{T_{n}}$ is $\mu_{T}^{n}$. Because $\mathbb{E}\left(\ln \left(a_{1}\right)\right)<0$, we have $\mathbb{P}-$ a.e $\lim _{n \rightarrow \infty} S_{n}=-\infty$ and moreover $\lim _{n \rightarrow \infty} T_{n}=\infty$ hence $\mathbb{P}-$ a.e, $\lim _{n \rightarrow \infty} S_{T_{n}}=-\infty$. We have that

$$
\mu_{T}^{n+1} * f(t)=\mathbb{E}\left(f\left(t-S_{T_{n+1}}\right)\right),
$$

and $\lim _{t \rightarrow \infty} f(t)=0$. So, using Lebesgue's theorem, we can conclude that $\lim _{n \rightarrow \infty} \mu_{T}^{n+1} * f(t)=0$

\section{Proof of lemma 2}

Lemma 2 us a direct consequence of the formula ${ }^{\alpha} \mu_{T}^{n} *{ }^{\alpha} h(t)=e^{\alpha t} \mu_{T}^{n} * h_{1}(t)$, Lemma 1 part 2 , and the fact that $h_{1}$ is non negative. 


\section{Proof of lemma 3}

The definition of ${ }^{\alpha} \mu^{\prime}$ and the condition $H_{\mu}(3)$ imply $\int|\ln (a(g))|^{\alpha} \mu^{\prime}(d g)<\infty$. The strict convexity of the function $\ln \int a^{s}(g) \mu(d g)(s>0)$ gives $\int \ln (a(g)){ }^{\alpha} \mu^{\prime}(d g)<0$.

It follows $\left.\int|\ln | b(g)\right|^{\alpha} \mu^{\prime}(d g)<\infty$.

As observed above, the existence and uniqueness of ${ }^{\alpha} \nu^{\prime}$ follows.

If $x_{0}$ is a fixed point of $\operatorname{supp}^{\alpha} \mu^{\prime}$ then for any $(a, b) \in \operatorname{supp} \mu$;

$a^{-1} x_{0}+b a^{-1}=x_{0}$, i.e $x_{0}(a-1)=b$.

This implies that $-x_{0}$ is a fixed point of supp $\mu$, which contradicts $H_{\mu}(4)$. Hence, as it well known (see [5]), ${ }^{\alpha} \nu^{\prime}$ has no atom.

In order to show ${ }^{\alpha} \mathbb{E}\left(T^{\prime}\right)<\infty$ we consider the space ${ }^{a} \Omega^{\#}=\mathbb{R} \times G^{\mathbb{Z}}$ and the extended bilateral shift defined by ${ }^{a} \theta(p, \omega)=\left(p_{1}, \theta \omega\right)$ where $p_{1}=g_{1}^{\prime}(p)$ and $\theta$ is the bilateral shift on $G^{\mathbb{Z}}$. We endow ${ }^{a} \Omega^{\#}$ with the Markov measure $\kappa^{\#}$ associated with the ${ }^{\alpha} Q$-invariant probability ${ }^{\alpha} \nu^{\prime}$. Clearly $\kappa^{\#}$ is ${ }^{a} \theta$-invariant and ergodic. Also we consider the fibered bilateral Markov chain $\left(p_{n}, V_{n}\right)$ on $\mathbb{R} \times \mathbb{R}^{*}$ where $V_{n}=p^{-1} p_{n}\left(a_{1} a_{2} \cdots a_{n}\right)=p^{-1}\left(p+R_{n}\right)$. Let $\tau$ be the first "ladder epoch" of $\left(p_{n}, V_{n}\right)$ (see [7]), i.e $\tau=\operatorname{Inf}\left\{n \geq 1 ; V_{n}>1\right\}$, hence $p^{-1} p_{\tau}>0$ and $\tau=T$ if $p>0$. We observe that the conditions in $H_{\mu}(3)$ implies $\int|p|^{\varepsilon \alpha} \nu^{\prime}(d p)<\infty$ for some $\varepsilon>0$, hence $\limsup _{|n| \rightarrow \infty} \frac{1}{|n|} \ell n\left|p_{n}\right| \leq 0$. Since ${ }^{\alpha} \mathbb{E}\left(\ln \left(a_{1}\right)\right)>0$ the ergodic theorem gives $\lim _{n \rightarrow \infty}\left|V_{n}\right|=\infty, \lim _{n \rightarrow-\infty}\left|V_{n}\right|=0$, in particular $\tau$ is finite $\kappa^{\#}-$ a.e. Since ${ }^{\alpha} \nu^{\prime}\left(\mathbb{R}_{+}\right)>0$ we can consider the Markov kernel ${ }^{\alpha} Q_{+}$induced by ${ }^{\alpha} Q$ on $\mathbb{R}_{+}$; the normalized restriction ${ }^{\alpha} \nu_{+}^{\prime}$ of ${ }^{\alpha} \nu^{\prime}$ to $\mathbb{R}_{+}$is ${ }^{\alpha} Q_{+}$-invariant and ergodic. We denote by ${ }^{a} \Omega_{+}^{\#}$ the subset of ${ }^{a} \Omega^{\#}$ defined by the conditions $p_{n}>0$ infinitely often for $n=n_{k}>0$ and $n=n_{-k}<0$. Since ${ }^{\alpha} \nu^{\prime}\left(\mathbb{R}_{+}\right)>0,{ }^{a} \Omega_{+}^{\#}$ has positive $\kappa^{\#}$-measure and we denote by $\kappa_{+}^{\#}$ the normalized restriction of $\kappa^{\#}$ to ${ }^{a} \Omega_{+}^{\#}$; then $\kappa_{+}^{\#}$ is invariant and ergodic under the corresponding induced shift ${ }^{a} \theta_{+}$. From above we know that $\lim _{k \rightarrow \infty} V_{n_{-k}}=0$, hence the time $\tau_{+}(\omega)=n_{-j}(j \geq 0)$, of the last strict maximum of $V_{n_{-k}}$ is finite $\kappa_{+}^{\#}-$ a.e. We define ${ }^{a} \Omega_{0}^{\#}=\left\{\sup _{k>0} V_{n_{-k}}<1\right\}=\left\{\tau_{+}=0\right\}$. Then we have ${ }^{a} \kappa_{+}^{\#}\left({ }^{a} \Omega_{0}^{\#}\right)>0$ since, by ${ }^{a} \theta_{+}$-invariance of $\kappa_{+}^{\#}$ :

$$
\begin{aligned}
& 1=\kappa_{+}^{\#}\left\{\tau_{+}>-\infty\right\}=\sum_{n \geq 0} \kappa_{+}^{\#}\left\{\tau_{+}=-n\right\} \leq \sum_{n \geq 0} \kappa_{+}^{\#}\left\{V_{-n}>\sup _{n_{-k}<-n} V_{n_{-k}}\right\}= \\
& \sum_{n \geq 0} \kappa_{+}^{\#}\left\{0>\sup _{k>0} V_{n_{-k}}\right\} \leq \infty \kappa_{+}^{\#}\left({ }^{a} \Omega_{0}^{\#}\right) .
\end{aligned}
$$

On the other hand, the definition of $\tau$ shows that for $\omega \in{ }^{a} \Omega_{0}^{\#}, \tau(\omega)$ is the first return time of ${ }^{a} \theta^{k}(\omega)$ to ${ }^{a} \Omega_{0}^{\#}$, so that ${ }^{a} \theta^{\tau}$ is the transformation of ${ }^{a} \Omega_{0}^{\#}$ induced by ${ }^{a} \theta$ on ${ }^{a} \Omega_{0}^{\#}$. Then Kac's theorem (see [23]) implies that ${ }^{a} \theta^{\tau}$ is ergodic with respect to the normalized restriction $\kappa_{0}^{\#}$ of $\kappa_{+}^{\#}$ to ${ }^{a} \Omega_{0}^{\#}$ and ${ }^{\alpha} \mathbb{E}(\tau)=\int \tau(\omega) \kappa_{0}^{\#}(d \omega)<\infty$. Also we denote by ${ }^{\alpha} \nu_{+}^{\tau}$ the push forward of $\kappa_{0}^{\#}$ to $\mathbb{R}_{+}$under the map $\omega \rightarrow p_{0}(\omega)$. Since the stopped kernel ${ }^{\alpha} Q_{+}^{\tau}$ and the map $\tau$ commute with $p_{0}$, the measure $\kappa_{+}^{\tau}$ is ${ }^{\alpha} Q_{+}^{\tau}$-invariant, ergodic and absolutely continuous with respect to ${ }^{\alpha} \nu_{+}$with ${ }^{\alpha} \mathbb{E}_{0}(\tau)={ }^{\alpha} \mathbb{E}(T)<\infty$. 


\section{Remark}

A different proof of $\alpha \mathbb{E}(T)<\infty$ uses the interpretation of $T=T^{\prime}$ as hitting time of the open set $\mathbb{R}_{+}$by the Markov chain with kernel ${ }^{\alpha} Q$ starting from 0 .

Since $\int a^{\delta}(g)^{\alpha} \mu^{\prime}(d g)<\infty, \int\left|b^{\delta}(g)\right|^{\alpha} \mu^{\prime}(d g)<\infty$ for $0<\delta<\alpha$, the operator defined by ${ }^{\alpha} Q$ on a space of Hölder functions on $\mathbb{R}$ (as in [13]) has a spectral gap. This implies ${ }^{\alpha} \mathbb{E}\left(T^{\prime}\right)<\infty$. The proof given above extends to the multidimensional case.

\section{IV - Appendix : a weak renewal theorem}

Proposition A Let $\left(Z_{n}\right)_{n \geq 1}$ a sequence of independant, identically distributed real random $\overline{\text { variables on } \mathbb{R}}$ with law $\eta$. Assume that $\int|z| \eta(d z)<+\infty$ and that $\gamma=\int z \eta(d z)>0$.

Let $\psi$ a bounded non negative Borel function which is supported on $[-a, a]$.

Then the potential $U \psi=\sum_{n=0}^{+\infty} \eta^{n} * \psi$ is a bounded function and we have

$$
\lim _{t \rightarrow+\infty} \frac{1}{t} \int_{0}^{t} U \psi(s) d s=\frac{1}{\gamma} \int_{\mathbb{R}} \psi(t) d t
$$

\section{$\underline{\text { Proof }}$}

If $\Sigma_{n}=\sum_{i=1}^{n} Z_{i}$, we have : $U \psi(s)=\sum_{n=0}^{+\infty} E\left[\psi\left(s-\Sigma_{n}\right)\right]$.

Because $\gamma=\int z \eta(d z)>0$ the random walk on $\mathbb{R}$ with law $\eta$ is transient and using the maximum principle we have that $\sup _{s \in \mathbb{R}} U \psi(s)<+\infty$.

For $\varepsilon>0, t>0$ denote

$$
\begin{aligned}
& n_{1}(t)=\left[\frac{1}{\gamma} \varepsilon t\right]=n_{1}, \quad n_{2}(t)=\left[\frac{1}{\gamma}(1-\varepsilon) t\right]=n_{2}, \\
& U_{n} \psi=\sum_{0}^{n-1} \eta^{k} * \psi, \quad U^{n} \psi=\sum_{n+1}^{\infty} \eta^{k} * \psi, \quad U_{n}^{m} \psi=\sum_{n}^{m} \eta^{k} * \psi .
\end{aligned}
$$

Then we have

where

$$
I(t)=\frac{1}{t} \int_{0}^{t} U \psi(s) d s=\sum_{1}^{3} I_{k}(t)-I_{4}(t)
$$

$$
\begin{array}{ll}
I_{1}(t)=\frac{1}{t} \int_{\mathbb{R}} U_{n_{1}}^{n_{2}} \psi(s) d s, & I_{2}(t)=\frac{1}{t} \int_{\mathbb{R}} U_{n_{1}} \psi(s) d s, \\
I_{3}(t)=\frac{1}{t} \int_{\mathbb{R}} U^{n_{2}} \psi(s) d s, & I_{4}(t)=\frac{1}{t} \int_{\mathbb{R}[0, t]} U_{n_{1}}^{n_{2}} \psi(s) d s .
\end{array}
$$

We have

$$
\begin{aligned}
& I_{1}(t)=\frac{n_{2}-n_{1}+1}{t}\left(\int_{\mathbb{R}} \psi(s) d s\right) \text { hence } \lim _{t \rightarrow+\infty} I_{1}(t)=\frac{(12 \varepsilon)}{\gamma} \int_{\mathbb{R}} \psi(s) d s, \\
& 0 \leq I_{4}(t) \leq\left(\frac{\left(n_{2}-n_{1}+1\right.}{t} \sup _{s \in \mathbb{R}}|\psi(s)|\right) \sup _{n_{1} \leq n \leq n_{2}}\left[\mathbb{P}\left(\Sigma_{n} \leq a\right)+\mathbb{P}\left(t-\Sigma_{n} \leq a\right)\right] .
\end{aligned}
$$

By the law of large numbers we know that $\mathbb{P}-$ a.e, $\lim _{n \rightarrow+\infty} \frac{\Sigma_{n}}{n}=\gamma>0$, hence :

$$
\lim _{t \rightarrow+\infty} \sup _{n_{1} \leq n \leq n_{2}}\left(\mathbb{P}\left\{\Sigma_{n} \leq a\right\}+P\left\{t-\Sigma_{n} \leq a\right\}\right)=0 .
$$

Hence $\lim _{t \rightarrow+\infty} I_{4}(t)=0$ and : $0 \leq \frac{I_{2}(t)}{t} \leq \frac{\varepsilon}{\gamma} \times \int \psi(s) d s$. 
Consider now $I_{3}(t)$ and denote for $n \in \mathbb{N}, s>0: \rho_{n}^{s}=\operatorname{Inf}\left\{k \geq n ;\left|V_{n}-s\right| \leq a\right\}$.

We use the interpretation of $U^{n} \psi$ as the expected number of visits to $\psi$ after time $n$ : $U^{n} \psi(x) \leq(U \psi) \mathbb{P}\left\{\rho_{n}^{s}<\infty\right\}$ with $n \frac{[(1+\varepsilon) t]}{\gamma}=n_{2}$, hence :

$$
I_{3}(t) \leq|U \psi| \mathbb{P}\left\{\Sigma_{k} \leq t+a \text { for some } k \geq \frac{(1+\varepsilon) t}{\gamma}\right\} .
$$

Since $\frac{\Sigma_{n}}{n}$ converges to $\gamma, \mathbb{P}-$ a.e, we get $\lim _{t \rightarrow \infty} I_{3}(t)=0$.

Since $\varepsilon$ is arbitrary we get finally : $\lim _{t \rightarrow \infty} I(t)=\frac{1}{\gamma} \int \psi(s) d s$.

\section{References}

[1] Babillot M., Bougerol P., Elie L. : The random difference equation $X_{n}=$ $A_{n} X_{n-1}+B_{n}$ in the critical case. Ann. Probab. 25,1 (478-493) (1997).

[2] Bardou F., Bouchaud J.P., Aspect A., Cohen-Tannoudji C. : "Levy Statistics and Laser Cooling". Cambridge Univ. Press (2002).

[3] Basrak B., Segers J. : Regularly varying time series. Stochastic Pocess Appl. 119, 1055-1080 (2009).

[4] Brofferio S., Buraczewski D., Damek E. : On the invariant measure of the random difference equation $X_{n}=A_{n} X_{n-1}+B_{n}$ in the critical case. Ann. I.H.P. $48,377-395$ (2012).

[5] Buraczewski D. and Damek E.,Guivarc'h Y., Hulanicki A., Urban R. : Tailhomogeneity of stationary measures for some multidimensional stochastic recursions. Probab. Theory Relat. Fields 145 n$^{\circ} 3-4$ 385-420 (2009).

[6] Dolgopyat D., Goldsheid I. : Quenched limit theorems for nearest neighbour random walks in 1D random environment. Comm. Math. Phys. 315, 1, 247-277 (2012).

[7] Feller W. : "An Introduction to Probability Theory and its Applications". vol. II, 2nd edn. Wiley New-York (1971).

[8] Galambos J. : The distribution of the largest coefficient in the continued fraction expansion. Quart. J. Math. 23, 147-151 (1972).

[9] Gnedenko B. : Sur la distribution limite d'une série aléatoire. Annals of Math. $44 \mathrm{n}^{\circ} 3,423-453$ (1943).

[10] Goldie C.M. : Implicit renewal theory and tails of solutions of random equations. Ann. Appl. Probab. 1(1) 126-166 (1991). 
[11] Guivarc'h Y. : Heavy tail properties of stationary solutions of multidimensional stochastic recursions. In "Dynamics and Stochastics". IMS Lecture Notes Monograph Series, vol. 48, pp 85-99. Institute of Mathematical Statistics, Beachwood (2006).

[12] Guivarc'h Y., Le Page E. : Homogeneity at infinity of stationary solutions of Multivariate Affine Stochastic Recursions. In "Random Matrices and Iterated Random functions". Alsmeyer G. - Löwe M. Eds. Springer Proceedings in Mathematics and Statistics 53, pp 119-135 (2013).

[13] Guivarc'h Y., Le Page E. : On spectral properties of a family of transfer operators and convergence to stable laws for affine random walks, Ergod. Th. Dynam. Sys. 28, 423-446 (2008).

[14] Guivarc'h Y., Le Page E. : Asymptotique des valeurs extrêmes pour les marches aléatoires affines. C.R.A.S (2013).

[15] Haan L., Resnick S.I., Rootzen H., Vries C.G. : Extremal behaviour of solutions to a stochastic difference equation with applications to $\mathrm{ARCH}$ processes. Stochastic Process. Appl. 32, 213-224 (1989).

[16] Kesten H. : Random difference equations and renewal theory for products of random matrices. Acta Mathematica 131, 207-248 (1973).

[17] Kesten H., Kozlov M., Spitzer F. : A limit law for random walk in a random environment, Compositio Math. 30 145-168 (1975).

[18] Kleinbock D.Y., Margulis G.A. : Logarithm laws for flows on homogeneous spaces, Inv. Math. 138, 451-494 (1999).

[19] Leadbetter M.R., Lindgren G., Rootzen H. : "Extremes and related properties of random sequences and processes", Springer, Berlin (1983).

[20] Pollicott M. : Limiting distributions for geodesic excursions on the modular surface, Contemp. Math. 469, 117-185 (2008).

[21] Solomon F. : Random walks in a random environment. Ann. Proba. 3, 1-31 (1975).

[22] Sullivan D. : Disjoint spheres, approximation by imaginary quadratic numbers and the logarithm law for geodesics, Acta Math. 149, 215-237 (1982).

[23] Wolfowitz J. : Remarks on the notion of recurrence. Bull A.M.S. 55, 394-395 (1949). 